\title{
Transformation of the Mechanical Properties of Fiber-Reinforced Plastic Tubes from the Cartesian Coordinate System into the Cylindrical Coordinate System for the Application of Bending Models
}

\author{
By Marco Siegl \\ Ingo Ehrlich
}

Fiber-reinforced plastic (FRP) tubes are used in many different industries, such as electrical engineering and pipeline construction. The tubes are frequently subjected to bending loads, depending on the application. In order that the dimensioning of the tubes can be ensured, analytical bending models are used to calculate the resulting stresses, strains and displacements in the individual layers of the laminate. This enables the making of a statement about the failure of the fiber-reinforced tube by choosing an appropriate failure criterion. For the use of these bending models, it is necessary to understand the respective underlying theory. The theory provides the basis for the mathematical description of the mechanical properties for a single-layered tube and using the relationships between the stresses and strains that occur in the Cylindrical coordinate system for this calculation step. For this reason, a redefinition of the compliance matrix from the transformation about the winding angle to the Cylindrical coordinate system and a modification of the stress and strain vectors is necessary, because the defined Cartesian coordinate system of the model cannot be used for wounded FRP tubes. The transformation causes an exchange of entries in the compliance matrix, which remain in the correct relationship between the particular stress and strains. This step is not specified and may lead to incorrect results due to the incorrect entry of compliances. The present publication refers to sketch on this issue and represent a simplification of the changeover to the level required by the bending models notation of vectors in the form of a permutation. In addition, a new name for the pre-acquisition of the redefined compliances is given to prevent confusion when entering the material law of a bending model. Finally, the permuted and redefined compliances are proved in an example to determine their accuracy.

Keywords: Bending model, Cylindrical coordinate system, Fiber-reinforced plastic tubes, Permutation of the compliances, Transformation of the compliances.

\footnotetext{
* PhD Student, Ostbayerische Technische Hochschule (OTH) Regensburg, Germany.

* Professor, Ostbayerische Technische Hochschule (OTH) Regensburg, Germany.
} 


\section{Introduction}

Fiber-reinforced plastics represent a major constituent in lightweight construction and are utilized increasingly in the industry due to their excellent properties. The specific strength and specific stiffness of FRPs exceed those of high-alloyed steels or light materials such as aluminum. FRP materials are mainly used in industries such as aviation and space technology, in which a weightminimization with simultaneous fulfillment of mechanical requirements is demanded for design goals. This leads to savings concerning the accelerating mass, thus allowing for greater drive power or payloads. This implies that FRPs are ideal lightweight materials for engineering components, which undergo acceleration or deceleration, which is the case in aerospace engineering, automotive and maritime engineering. In addition, dynamically moving components in general engineering applications can be produced with this lightweight construction to energetically optimize machines.

Due to the high chemical resistance, FRPs are also used for equipment and pipeline construction in corrosive environment applications. The non-conducting properties of glass fibers enables the use of glass-fiber-reinforced plastics (GFRP) as insulators and switches in the electrical industry. In many of these industrial sectors, axially symmetric structures made of FRP are used. For example, a fluid has to flow through or a machine operates in the interior and has to be sealed with gas. Furthermore, these tubular structures are subjected to bending caused by e. g. wind loads or module-specific design elements. The calculation of the stresses and deformations of FRPs under the described bending load is very challenging because of the anisotropy of the material and the multi-layered structure of the composite. Nevertheless, there are some calculation models for fiber-reinforced tubes, which are based on the definition of the linear-elastic material properties of a single layer and the associated coordinate system.

After this step, the mathematical theory of cylindrical anisotropic elasticity by Lekhnitskii (1963) can be specified. This includes the set-up of the basic equations for the strain-displacement relations for a tube with an orthotropic single layer, an indication of the stress relationships and their equilibrium conditions. In addition to that, two systems from the relationships and material properties are generated in the form of partial differential equations over stress functions through whether the bending problem or the tensile, torsion and pressure problem can be solved. Jolicoeur and Cardou (1994) established a general analytical solution based on the work of Lekhnitskii (1963) for stresses and displacements of a multi-layered tube, in which the individual layers are composed of orthotropic material and the boundary conditions between the individual layers and on the outer surfaces are defined. They also noted a deformation of the circular cross section due to bending stress, for which reason the Bernoulli-Euler hypothesis is strictly not allowed to be used for FRP cylinders. Chouchaoui and Ochoa (1999) used similar constitutive equations as Jolicoeur and Cardou (1994), but they considered only perfect bonding between the layers in comparison to Jolicoeur and Cardou (1994), who differentiated slip and friction as boundary condition. Both publications are predicted on the 
assumption that the loading case for bending is defined with two moments acting at the ends of the tube, so that a constant curvature is resulting along the longitudinal tube axis. Therefore the equivalent flexural stiffness $\langle E I\rangle$ for the multi-layered tube can be calculated. Tarn and Wang (2001) presented a state space approach to the bending of the laminated composite tubes. Here, the system matrix in the Cylindrical coordinate system is independent of the radius $r$, because of a judicious arrangement of the displacement and stress variables. Thus, the calculation process is followed by a reduction of the large system of equations for the stress and displacement expressions. Derisi (2008) used the flexural stiffness to describe a cantilever beam of FRP with a transverse force at the free end to calculate the deflection. Shadmehri et al. (2011) exhibited another way for defining the equivalent bending stiffness $\langle E I\rangle$, which is a function of the radius $r$ and the laminate stiffness coefficients for anisotropic materials. In addition to the described warping of the cross section by Jolicoeur and Cardou (1994), also warping inhibition, transverse shear deformation and non-uniform twist on composite tubes occurs. Due to these effects, therein the referenced non-classical composite beam theory is used to define the equivalent bending stiffness $\langle E I\rangle$. Derisi et al. (2012) also used the non-classical laminate theory to define the equivalent bending stiffness $\langle E I\rangle$, because the system of equations would be large for many layers. Instead, this investigation compared the flexural stiffness of three-point-bending tests for three different composite tubes with an analogous aluminum tube and the calculated flexural stiffness. A new simulation technique was also presented and has been proved with experimental results. Geuchy Ahmad and Hoa (2016) validated the analytical equivalent flexural stiffness $\langle E I\rangle$ according to Jolicoeur and Cardou (1994) with experiments on two thick walled composite tubes. They used a pure bending test setup without concentrated loading points in the force introduction as in three- or four-point bending tests, which is developed by Shadmehri (2012). Here, the tube is fixed inside the bending facility with low melting point alloy. To realize the required bending moment at each end of the tube, the construction includes two hydraulic cylinders and moment-arm assemblies. The deformation behavior was measured with strain gauges at different locations of the tube and speckle pattern to exploit the surface deformation also with a Digital Image Correlation measurement system. The experimental results correlate to the analytic bending stiffness by Jolicoeur and Cardou (1994), but with only two specimens the experiment does not provide an adequate statistical coverage. Sarvestani et al. (2016) presented a new high-order displacement-based method for thick cantilever tubes under transverse loading, which has a good match with the experimental data, which are generated with a three-point bending test, FEM and the Lekhnitskii (1963) solution with a $\left[0^{\circ}\right]$ composite tube, because Lekhnitskii only examines single layer cylinders with monolithic homogeneous orthotropic cylindrical shells. The method was also based on the equation of Lekhnitskii (1963), but they used a layer-wise theory with Lagrangian linear interpolation functions, because of the thick composite tubes, and using the principle of minimum total potential energy to get the equilibrium equations of a laminated orthotropic straight tube. 


\section{Analysis}

A correct material definition requires a strict distinction between the Cartesian, the Cylindrical coordinate system and the coordinate system that defines the external loads. A unidirectional (UD) composite layer is also characterized by a local Cartesian coordinate system and forms the basis for a mechanical description of the anisotropic material properties. According to a transformation in the Cartesian and the transformation into the Cylindrical coordinate system a new defined permutation matrix is established. The reason is the deviant definition of the Cartesian coordinate system by Lekhnitskii (1963). After transformation, the corresponding bending models can finally be applied.

\section{Unidirectional Layer}

The unidirectional single layer consists of quasi-endless long reinforced fibers imbedded in a matrix system. In case of FRP the matrix is a plastic type. The 1direction of the local 123-coordinate system is congruent with the fiber direction (see Figure 1). The transverse directions are indicated by the alignment of the layer. For transversely isotropic materials the 3-direction is the thickness direction of the single-layer and 2-direction stretched with the 1-direction a plane, in which the fibers may be displayed in their entire length.

Figure 1. Volume Element of a Unidirectional Fiber-reinforced Layer with Associated Coordinate System and Spatial State of Stress (Schober, 2008)

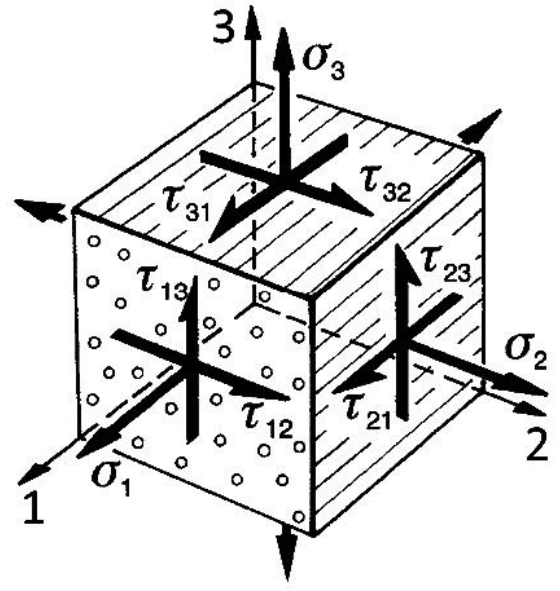

Figure 1 shows the stresses and their directions on the volume element. Thus, the stresses are clearly marked, two indices are given by the Anglo-Saxon principle. The first index indicates the direction of the normal line of the plane of action to which the stress is applied. The second index indicates the direction of the stresses. A shear stress is defined as positive when the stress extends in the positive axis direction at its positive sectional plane. The three-dimensional stress tensor of the volume element of Figure 1 is given by 


$$
[\sigma]=\left[\begin{array}{ccc}
\sigma_{1} & \tau_{12} & \tau_{13} \\
\tau_{21} & \sigma_{2} & \tau_{23} \\
\tau_{31} & \tau_{32} & \sigma_{3}
\end{array}\right]=\left[\begin{array}{ccc}
\sigma_{1} & \tau_{12} & \tau_{13} \\
& \sigma_{2} & \tau_{23} \\
\text { sym. } & & \sigma_{3}
\end{array}\right]
$$

The moment equilibrium at the volume element is described by the relations $\tau_{12}=\tau_{21}, \tau_{13}=\tau_{31}$ and $\tau_{23}=\tau_{32}$, whereby the stress tensor is symmetric. The stress tensor can be written depending on the Voigt's notation (Voigt 1966) as a $6 \times 1$ vector

$$
\{\sigma\}=\left\{\begin{array}{c}
\sigma_{1} \\
\sigma_{2} \\
\sigma_{3} \\
\tau_{23} \\
\tau_{13} \\
\tau_{12}
\end{array}\right\} .
$$

Using this notation for the stress vector $\{\sigma\}$ and for the strain vector $\{\varepsilon\}$, the constitutive equation according to Hooke's law for transversely isotropic unidirectional single layer results to

$$
\begin{aligned}
& \left\{\begin{array}{c}
\sigma_{1} \\
\sigma_{2} \\
\sigma_{3} \\
\tau_{23} \\
\tau_{13} \\
\tau_{12}
\end{array}\right\}=\left[\begin{array}{cccccc}
C_{11} & C_{12} & C_{13} & 0 & 0 & 0 \\
& C_{22} & C_{23} & 0 & 0 & 0 \\
& & C_{33} & 0 & 0 & 0 \\
& & & C_{44} & 0 & 0 \\
& & \text { sym. } & & C_{55} & 0 \\
& & & & & C_{66}
\end{array}\right] \cdot\left\{\begin{array}{c}
\varepsilon_{1} \\
\varepsilon_{2} \\
\varepsilon_{3} \\
\gamma_{23} \\
\gamma_{13} \\
\gamma_{12}
\end{array}\right\}, \\
& \{\sigma\}=[C] \cdot\{\varepsilon\}
\end{aligned}
$$

and

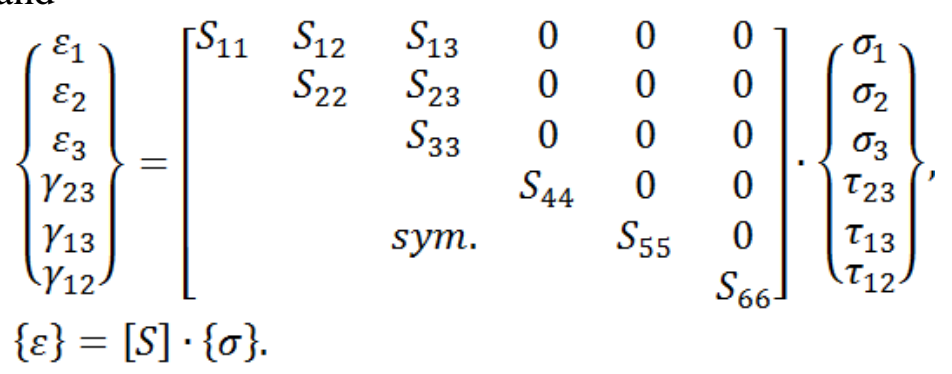

The compliance matrix $[S]$, which is necessary for further calculation, has nine independent elastic constants, which are expressed by the respective engineering constants 


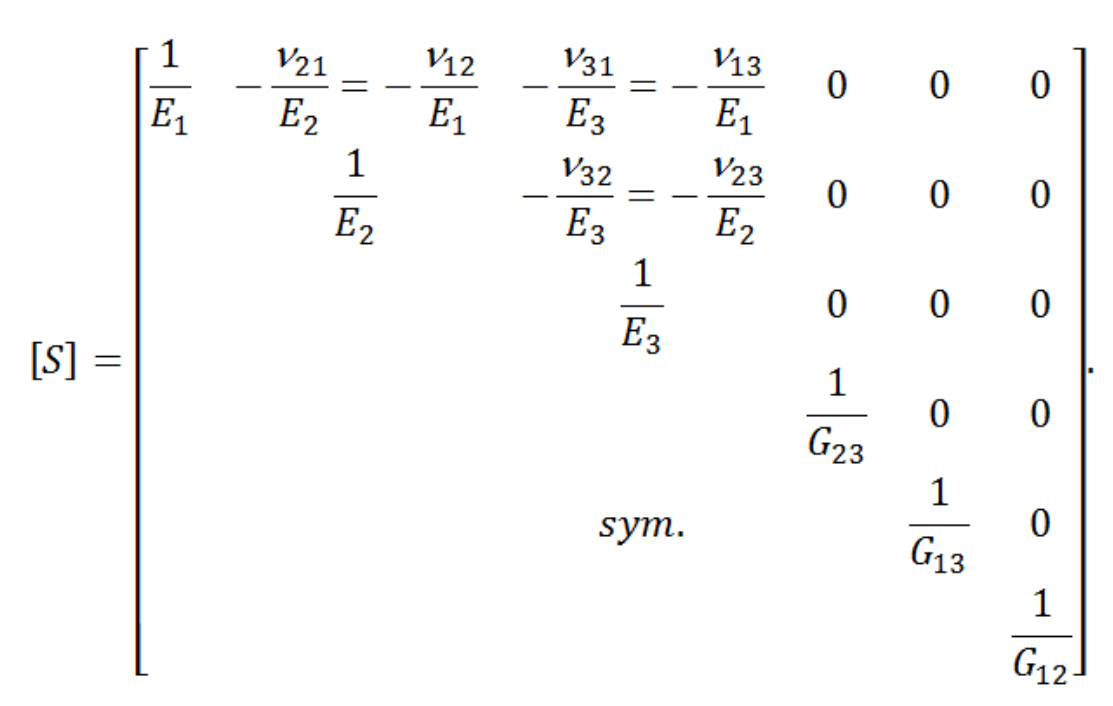

The compliances $S_{12}, S_{13}$ and $S_{23}$ in equation (5) are indicated by the Maxwell-Betti Reciprocal Relations (Ehrenstein, 2006; Ehrlich, 2004; Schürmann, 2007). The stiffness matrix $[C]$ is obtained by inverting the compliance matrix $[S]$. Here, it's possible to mistakenly interchange the compliances in equation (5) with the used compliances of Chouchaoui and Ochoa (1999), because they specified the compliances with $C_{i j}$ and the respective compliances are not expressed in terms of the elastic constants as in equation (5).

In individual unidirectional layers of FRPs the special case of orthotropic, transverse isotropy, occurs. Thereby, an infinite number of planes of symmetry with a rotation around the longitudinal 1-axis (see Figure 1) are present. As a result, the entries in the stiffness and compliance matrix are not reduced, but comprise only five independent elastic constants because the material properties transverse to the fiber direction are isotropic (Ehrenstein, 2006; Ehrlich, 2004; Moser, 1992; Schürmann, 2007). Hence, the following relations can be specified for the engineering constants in the transverse direction

$$
E_{2}=E_{3}, \quad v_{12}=v_{13}, \quad v_{23}=v_{32}, \quad G_{12}=G_{13}, \quad G_{23}=(6)
$$

These relations can be used for a single layer in the compliance matrix in equation (5).

\section{Cartesian Coordinate System}

The bending models are designed for axially symmetric structures such as tubes. Thus, it is beneficial to define the global Cartesian coordinate system on the basis of a tube. Due to the axisymmetric material properties of a coiled tubing layer of FRP material, the coordinate system is placed on the surface of the layer (see Figure 2). In Figure 2 a single-layered composite tube with wound fibers with orientation to the xyz-coordinate system of the angle $\alpha$ is illustrated. The $\mathrm{x}$-axis is 
the longitudinal axis of the tube. The y-axis is always directed tangentially to the FRP tube. The $\mathrm{z}$-axis is orthogonal to the laminate surface with an orientation towards the outside of the composite.

Figure 2. Cartesian Coordinate System for the Transformation of the Material Properties

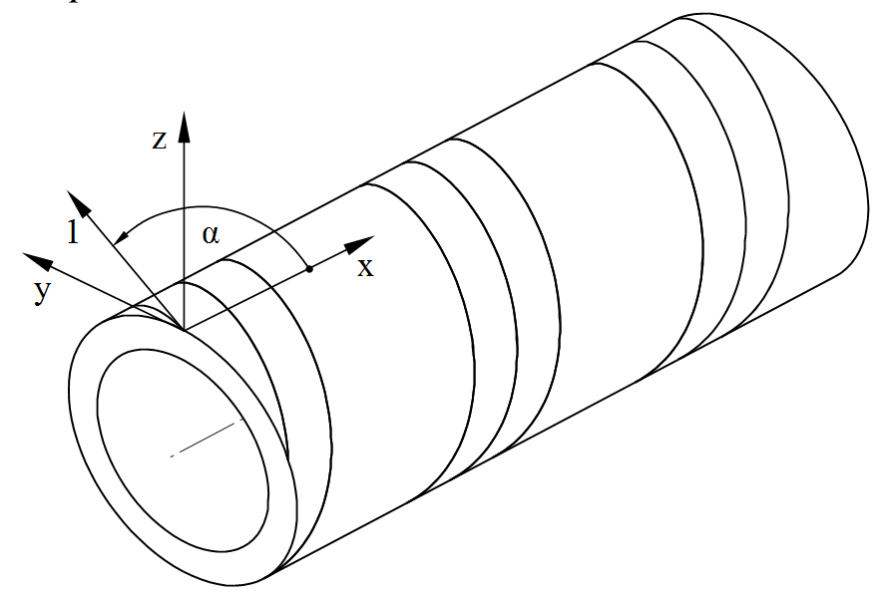

By reason of the mechanical design of a fiber-reinforced plastic tube under certain loads, an orientation of the fibers at a certain winding angle $\alpha$ is necessary. The direction of the angle and the definition of local and global coordinate systems to each other is shown in Figure 3. The 1-direction is maintained in the fiber longitudinal direction, whereas the local 2-direction is perpendicular to this. The angle $\alpha$ is defined in the mathematical positive direction from the global $\mathrm{x}$ axis to the local 1-axis (Schürmann, 2007).

Figure 3. 2D-Transformation of the Material Properties from the Local Coordinate System into the Global Coordinate System

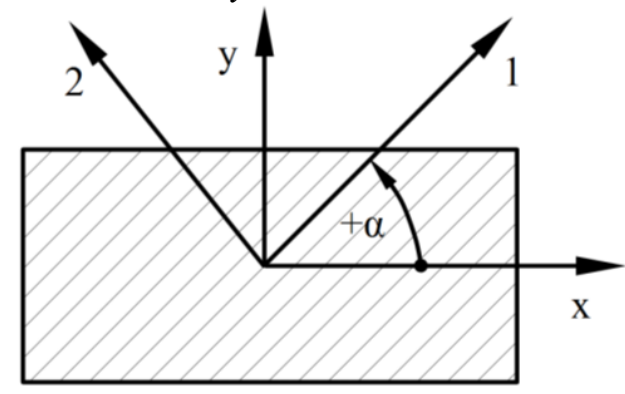

Thus, the global strains $\{\varepsilon\}_{x y z}$ in dependence of the winding angle $\alpha$ and the global tensions $\{\sigma\}_{x y z}$ for the tube can be specified. Therefore, the compliance matrix $[S]$ has to be converted to the transformed compliance matrix $[\bar{S}]$ as

$$
[\bar{S}]=\left[T_{\sigma}\right]_{3 D}^{T}[S]\left[T_{\sigma}\right]_{3 D},
$$


with the $3 \mathrm{D}$ transformation matrix $\left[T_{\sigma}\right]_{3 D}$ for a rotation about the $\mathrm{z}$-axis (Schürmann, 2007). This is useful because the assignment of the fiber direction is ensured to the coordinates. The $3 \mathrm{D}$ transformation matrix $\left[T_{\sigma}\right]_{3 D}$ is defined

$$
\left[T_{\sigma}\right]_{3 D}=\left[\begin{array}{cccccc}
\cos ^{2} \alpha & \sin ^{2} \alpha & 0 & 0 & 0 & 2 \sin \alpha \cos \alpha \\
\sin ^{2} \alpha & \cos ^{2} \alpha & 0 & 0 & 0 & -2 \sin \alpha \cos \alpha \\
0 & 0 & 1 & 0 & 0 & 0 \\
0 & 0 & 0 & \cos \alpha & -\sin \alpha & 0 \\
0 & 0 & 0 & \sin \alpha & \cos \alpha & 0 \\
-\sin \alpha \cos \alpha & \sin \alpha \cos \alpha & 0 & 0 & 0 & \cos ^{2} \alpha-\sin ^{2} \alpha
\end{array}\right]
$$

and $\left[T_{\sigma}\right]_{3 D}^{T}$ is the transpose matrix of the $3 \mathrm{D}$ transformation matrix $\left[T_{\sigma}\right]_{3 D}$ of the stresses (Altenbach et al., 2004; Ehrlich, 2004).

At a winding angle of $0^{\circ}<\alpha<90^{\circ}$, a monotropic material behavior for a single layer is resulting, which is expressed according to Schürmann (2007) in the constitutive equation (9)

$$
\left\{\begin{array}{c}
\varepsilon_{x} \\
\varepsilon_{y} \\
\varepsilon_{z} \\
\gamma_{y z} \\
\gamma_{x z} \\
\gamma_{x y}
\end{array}\right\}=\left[\begin{array}{cccccc}
\overline{S_{11}} & \overline{S_{12}} & \overline{S_{13}} & 0 & 0 & \overline{S_{16}} \\
& \overline{S_{22}} & \overline{S_{23}} & 0 & 0 & \overline{S_{26}} \\
& & \overline{S_{33}} & 0 & 0 & \overline{S_{36}} \\
& & & \overline{S_{44}} & \overline{S_{45}} & 0 \\
& & \text { sym. } & & \overline{S_{55}} & 0 \\
\tau_{y z}
\end{array}\right\} \cdot\left\{\begin{array}{c}
\sigma_{x} \\
\sigma_{y} \\
\sigma_{z} \\
\tau_{y z} \\
\tau_{x y}
\end{array}\right\},
$$

\section{Cylindrical Coordinate System}

For the use of the relations of Lekhnitskii (1963) it is essential to transform the material behavior into a Cylindrical coordinate system. This is realized by a redefinition of the Cartesian axes of Figure 2 in the Cylindrical coordinates in Figure 4. However, the transformation with the prevalent used Cartesian coordinate system in Figure 2 is not equal to the coordinate system transformation by Lekhnitskii (1963), who defined the longitudinal tube axis with $\mathrm{z}$ and remained constant during the transformation process. The reason for further assignment of the Cartesian coordinate system as in Figure 2 is the description of the material behavior. For the associated transformation a rotation around the z-axis, shown in Figures 2 and 3, is defined. Opposed to Lekhnitskii (1963) the x-axis defines the longitudinal pipe axis.

The tube longitudinal $\mathrm{x}$-axis in the Cartesian coordinate system is the $\tilde{z}$-axis of the Cylindrical coordinate system. The tilde over the $\mathrm{z}$ serves to ensure that no confusion occurs with the $\mathrm{z}$-axis of the Cartesian coordinate system. The equations of Lekhnitskii (1963) are indicated by $\mathrm{z}$, so the corresponding parameters with $\tilde{z}$ should be used here. In the Cylindrical coordinate system, the $\mathrm{z}$-axis corresponds to the radius $r$, which is always orthogonal to the laminate surface. The circumferential angle $\Theta$ correlates to the $y$-axis and is oriented by a 
clockwise rotation over the $\tilde{z}$-axis. This is due to the original xyz-coordinate system the rotation is around the $\mathrm{x}$-axis and the rotation direction is defined from the positive $y$-axis to the positive $z$-axis.

Figure 4. Cylindrical Coordinate System to Describe the Stress-strain Relationships of the Fiber-reinforced Plastic Tube

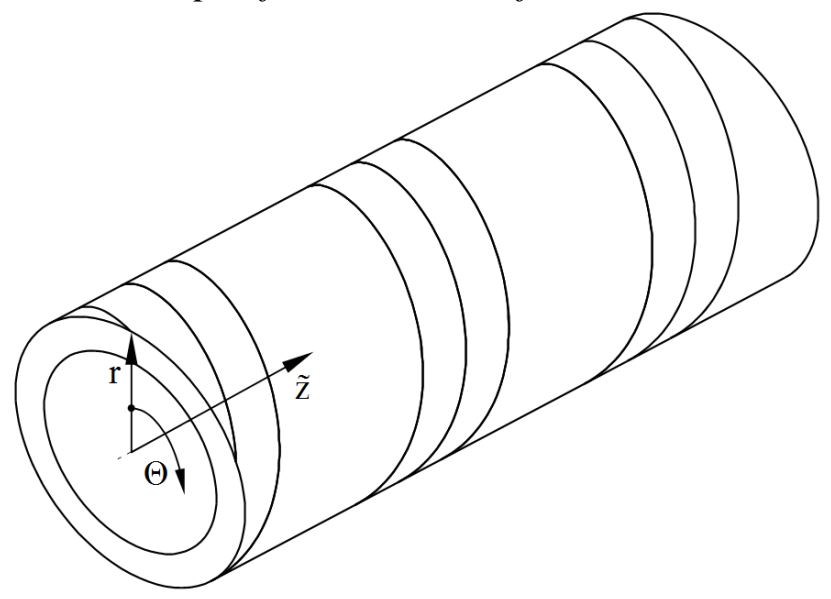

The stress tensor in the Cylindrical coordinate system according to Figure 4 equates to equation (1) with

$$
[\sigma]_{c y l}=\left[\begin{array}{ccc}
\sigma_{\tilde{z}} & \tau_{\tilde{z} \theta} & \tau_{\tilde{z} r} \\
\tau_{\theta \tilde{z}} & \sigma_{\theta} & \tau_{\theta r} \\
\tau_{r \tilde{z}} & \tau_{r \theta} & \sigma_{r}
\end{array}\right] .
$$

The stress tensor in cylindrically coordinates can be written as a $6 \times 1$ vector depending on the Voigt's notation (Voigt 1966)

$$
\{\sigma\}_{c y l}=\left\{\begin{array}{c}
\sigma_{\tilde{z}} \\
\sigma_{\theta} \\
\sigma_{r} \\
\tau_{\theta r} \\
\tau_{\tilde{z} r} \\
\tau_{\tilde{z} \theta}
\end{array}\right\}
$$

and the strain vector $\{\varepsilon\}_{\text {cyl }}$ applies to the same indexing. According to equation (9), the constitutive equation in Cylindrical coordinate system is

$$
\left\{\begin{array}{c}
\varepsilon_{\tilde{z}} \\
\varepsilon_{\theta} \\
\varepsilon_{r} \\
\gamma_{\theta r} \\
\gamma_{\tilde{z} r} \\
\gamma_{\tilde{z} \theta}
\end{array}\right\}=\left[\begin{array}{cccccc}
\overline{S_{11}} & \overline{S_{12}} & \overline{S_{13}} & 0 & 0 & \overline{S_{16}} \\
& \overline{S_{22}} & \overline{S_{23}} & 0 & 0 & \overline{S_{26}} \\
& & \overline{S_{33}} & 0 & 0 & \overline{S_{36}} \\
& & & \overline{S_{44}} & \overline{S_{45}} & 0 \\
& & \text { sym. } & & \overline{S_{55}} & 0 \\
& & & & & \overline{S_{66}}
\end{array}\right] \cdot\left\{\begin{array}{c}
\sigma_{\tilde{z}} \\
\sigma_{\theta} \\
\sigma_{r} \\
\tau_{\theta r} \\
\tau_{\tilde{z} r} \\
\tau_{\tilde{z} \theta}
\end{array}\right\},
$$




$$
\{\varepsilon\}_{c y l}=[\bar{S}] \cdot\{\sigma\}_{c y l} .
$$

But the stress and strain vector entries are in a different order due to the definition of the $\mathrm{x}$-axis as the radius $r$ and the $\mathrm{z}$-axis as longitudinal axis for the coordinate transformation according to Lekhnitskii (1963). The stress tensor $[\sigma]_{c y l, L}$ by Lekhnitskii (1963) is defined as

$$
[\sigma]_{c y l, L}=\left[\begin{array}{ccc}
\sigma_{r} & \tau_{r \theta} & \tau_{r \tilde{z}} \\
\tau_{\theta r} & \sigma_{\theta} & \tau_{\theta \tilde{z}} \\
\tau_{\tilde{z} r} & \tau_{\tilde{z} \theta} & \sigma_{\tilde{z}}
\end{array}\right]
$$

and therefore the constitutive equation in the Cylindrical coordinate system

$$
\begin{aligned}
& \left\{\begin{array}{c}
\varepsilon_{r} \\
\varepsilon_{\theta} \\
\varepsilon_{\tilde{z}} \\
\gamma_{\theta \tilde{z}} \\
\gamma_{r \tilde{z}} \\
\gamma_{r \theta}
\end{array}\right\}=\left[\begin{array}{cccccc}
\overline{L_{11}} & \overline{L_{12}} & \overline{L_{13}} & \overline{L_{14}} & 0 & 0 \\
& \overline{L_{22}} & \overline{L_{23}} & \overline{L_{24}} & 0 & 0 \\
& & \overline{L_{33}} & \overline{L_{34}} & 0 & 0 \\
& & & \overline{L_{44}} & 0 & 0 \\
& & \text { sym. } & & \overline{L_{55}} & \overline{L_{56}} \\
\{\varepsilon\}_{c y l, L} & =[\bar{L}] \cdot\{\sigma\}_{c y l, L}
\end{array}\right] \cdot\left\{\begin{array}{c}
\sigma_{r} \\
\sigma_{\theta} \\
\sigma_{\tilde{z}} \\
\tau_{\theta \tilde{z}} \\
\tau_{r \tilde{z}} \\
\tau_{r \theta}
\end{array}\right\},
\end{aligned}
$$

with the new nomenclature for the transformed compliance matrix to Lekhnitskii $[\bar{L}]$. This renaming is necessary, because on the one hand, for instance, Jolicoeur and Cardou (1994) indicated the transformed compliances by $C_{i j}$ and Lekhnitskii (1963) specified them by $a_{i j}$. On the other hand, the indices only specify the space in the matrix and not the actual transformed compliances according to equation (12). However, to define the compliance matrix $[\bar{L}]$ a changeover of the transformed compliance matrix $[\bar{S}]$ of equation (12) in the relationship of equation (14) is necessary. In equation (15) the compliance matrix, with the indexing according to equation (12) related to the stress and strain vector notation by Lekhnistskii (1963), is demonstrated.

$$
\left\{\begin{array}{c}
\varepsilon_{r} \\
\varepsilon_{\theta} \\
\varepsilon_{\tilde{z}} \\
\gamma_{\theta \tilde{z}} \\
\gamma_{\gamma_{\tilde{z}} \tilde{z}} \\
\gamma_{r \theta}
\end{array}\right\}=\left[\begin{array}{cccccc}
\overline{S_{33}} & \overline{S_{32}} & \overline{S_{31}} & \overline{S_{36}} & 0 & 0 \\
& \overline{S_{22}} & \overline{S_{21}} & \overline{S_{26}} & 0 & 0 \\
& & \overline{S_{11}} & \overline{S_{16}} & 0 & 0 \\
& & & \overline{S_{66}} & 0 & 0 \\
& & \text { sym. } & & \overline{S_{55}} & \overline{S_{54}} \\
& & & & & \overline{S_{44}}
\end{array}\right] \cdot\left\{\begin{array}{c}
\sigma_{r} \\
\sigma_{\theta} \\
\sigma_{\tilde{z}} \\
\tau_{\theta \tilde{z}} \\
\tau_{r \tilde{z}} \\
\tau_{r \theta}
\end{array}\right\},
$$

wherefore the definition of $[\bar{L}]$ is 


$$
[\bar{L}]=\left[\begin{array}{cccccc}
\overline{L_{11}} & \overline{L_{12}} & \overline{L_{13}} & \overline{L_{14}} & 0 & 0 \\
& \overline{L_{22}} & \overline{L_{23}} & \overline{L_{24}} & 0 & 0 \\
& & \overline{L_{33}} & \overline{L_{34}} & 0 & 0 \\
& & & \overline{L_{44}} & 0 & 0 \\
& & \text { sym. } & & \overline{L_{55}} & \frac{1 L_{56}}{L_{56}}
\end{array}\right]=\left[\begin{array}{cccccc}
\overline{S_{33}} & \overline{S_{32}} & \overline{S_{31}} & \overline{S_{36}} & 0 & 0 \\
& \overline{S_{22}} & \overline{S_{21}} & \overline{S_{26}} & 0 & 0 \\
& & \overline{S_{11}} & \overline{S_{16}} & 0 & 0 \\
& & & \overline{S_{66}} & \frac{0}{1} & 0 \\
& & \text { sym. } & & \overline{S_{55}} & \frac{S_{54}}{S_{44}}
\end{array}\right] .
$$

The changeover to the transformed compliance matrix $[\bar{L}]$ can be realized by exchanging the first and third as well as the fourth and sixth row and column while retaining the respective elasticity relations and taking into account the indexing of the stresses and the strains in the Cylindrical coordinate system like in equation (14). Therefore, it is necessary to establish a permutation matrix $[P]$ that performs this changeover process

$$
[P]=\left[\begin{array}{llllll}
0 & 0 & 1 & 0 & 0 & 0 \\
0 & 1 & 0 & 0 & 0 & 0 \\
1 & 0 & 0 & 0 & 0 & 0 \\
0 & 0 & 0 & 0 & 0 & 1 \\
0 & 0 & 0 & 0 & 1 & 0 \\
0 & 0 & 0 & 1 & 0 & 0
\end{array}\right]
$$

The permutation matrix $[P]$ is formed from the identity matrix $[E]$ and the exchange of the required line is realized by shifting the element 1 in the new row/column from the main diagonal. For row-interchanges, the permutation matrix $[P]$ must be included in the multiplication before the transformed-toexchange compliance matrix $[\bar{S}]$. For a column-exchange, the permutation $[P]$ is subsequently inserted (Schmidt and Trenkler, 2015). Hence, the cylindrical permuted compliance matrix $[\bar{L}]$ for the calculation according to Lekhnitskii (1963) results to

$$
[\bar{L}]=[P][\bar{S}][P] .
$$

Considering the two equations (16) and (18) and Lekhnitskii (1963) the reduced elastic constants $\beta_{i j}$ for the bending models can be calculated with

$$
\beta_{i j}=\overline{L_{l j}}-\frac{\overline{L_{l 3}} \overline{L_{3 j}}}{\overline{L_{33}}}
$$

According to Chouchaoui and Ochoa (1999) all reduced constants with the following indices are

$$
\beta_{i 3}=\beta_{3 j}=0
$$

and finally the reduced elastic constant matrix $[\beta]$ is 


$$
[\beta]=\left[\begin{array}{cccccc}
\beta_{11} & \beta_{12} & 0 & \beta_{14} & 0 & 0 \\
\beta_{21} & \beta_{22} & 0 & \beta_{24} & 0 & 0 \\
0 & 0 & 0 & 0 & 0 & 0 \\
\beta_{41} & \beta_{42} & 0 & \beta_{44} & 0 & 0 \\
0 & 0 & 0 & 0 & \beta_{55} & \beta_{56} \\
0 & 0 & 0 & 0 & \beta_{65} & \beta_{66}
\end{array}\right] .
$$

\section{Example}

To illustrate the permutation, an example with material properties of GFRPs will be presented. At first, the material properties are defined and transformed with the winding angle $\alpha$. Finally, the changeover of compliances in the Cylindrical coordinate system with the permutation matrix is presented. The material values are shown in tendered form, so that the calculation method can be comprehended. In reference to literature values (Ehrenstein 2006, Moser 1992) a single-layered tube is defined by the material properties of a glass fiber and epoxy resin in Table 1. Glass fibers and epoxy resin both have isotropic material properties.

The homogenization of the properties of the fiber and the matrix to the material characteristics of a unidirectional single layer are given with each applied rule of mixtures in Table 2.

Table 1. Material Properties of a Glass-fiber-reinforced Plastic with Epoxy Resin (Ehrenstein, 2006; Moser, 1992)

\begin{tabular}{|c|c|c|}
\hline & Material Properties & Quantification \\
\hline \multirow{4}{*}{$\begin{array}{l}\dot{\bar{\nu}} \\
\overrightarrow{\mid \vec{I}} \\
\tilde{n} \\
\tilde{\Xi} \\
\overrightarrow{0}\end{array}$} & Longitudinal modulus of elasticity & $E_{f}=73000 \mathrm{MPa}$ \\
\hline & Transverse modulus of elasticity & $E_{f 2}=E_{f}=73000 \mathrm{MPa}$ \\
\hline & Poisson's ratio & $v_{f}=0.25$ \\
\hline & Shear modulus & $G_{f}=\frac{E_{f}}{2\left(1+v_{f}\right)}=29200 \mathrm{MPa}$ \\
\hline \multirow{4}{*}{ 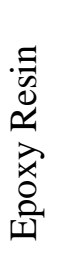 } & Longitudinal modulus of elasticity & $E_{m}=3500 \mathrm{MPa}$ \\
\hline & Transverse modulus of elasticity & $E_{m 2}=E_{m}=3500 \mathrm{MPa}$ \\
\hline & Poisson's ratio & $v_{m}=0.35$ \\
\hline & Shear modulus & $G_{m}=\frac{E_{m}}{2\left(1+v_{m}\right)}=1296 \mathrm{MPa}$ \\
\hline & Fiber volume content (ideal) & $\varphi_{f}=0.6$ \\
\hline
\end{tabular}


Table 2. Homogenization of the Unidirectional Layer

\begin{tabular}{ll}
\hline Material Properties & Quantification \\
\hline $\begin{array}{l}\text { Longitudinal modulus of elasticity according } \\
\text { to Moser (1992) }\end{array}$ & $E_{1}=45216 \mathrm{MPa}$ \\
$\begin{array}{l}\text { Transverse modulus of elasticity according to } \\
\text { Puck (1967) }\end{array}$ & $E_{2}=14845 \mathrm{MPa}$ \\
$\begin{array}{l}\text { Longitudinal-Transverse Shear modulus } \\
\text { according to Förster and Knappe (1971) }\end{array}$ & $G_{12}=5825 \mathrm{MPa}$ \\
$\begin{array}{l}\text { Poisson's ratio according to Jones (1999) } \\
\text { Poisson's ratio with the Maxwell-Betti }\end{array}$ & $v_{12}=0.29$ \\
$\begin{array}{l}\text { Reciprocal Relation } \\
\text { Poisson's ratio according to Foye (1972) }\end{array}$ & $v_{21}=v_{12} \frac{E_{2}}{E_{1}}=0.095$ \\
$\begin{array}{l}\text { Transverse-Transverse shear modulus } \\
\text { (Transversely isotropic UD-layer) }\end{array}$ & $v_{23}=0.36$ \\
\hline
\end{tabular}

A fiber-reinforced UD layer has a transversely isotropic material behavior, and therefore all properties in the transverse direction are equal. The material properties in Table 2 and the equation (5) result in the compliance matrix

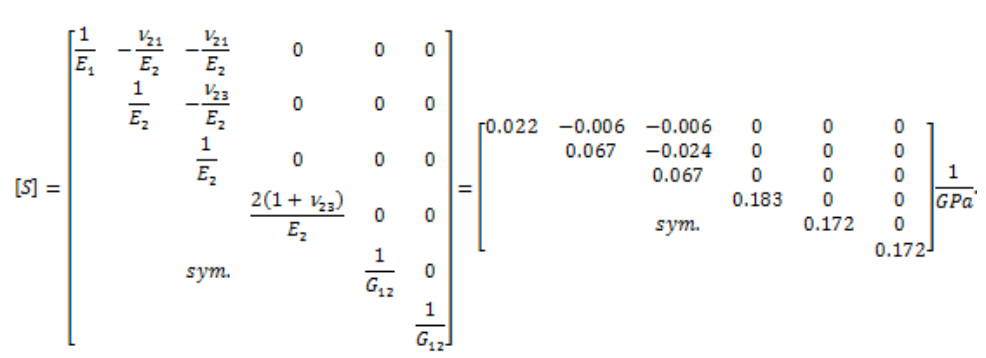

The following transformed compliance matrix in the Cartesian coordinate system results in connection with the equations (7), (8) and (9) for a winding angle of $\alpha=60^{\circ}$ in

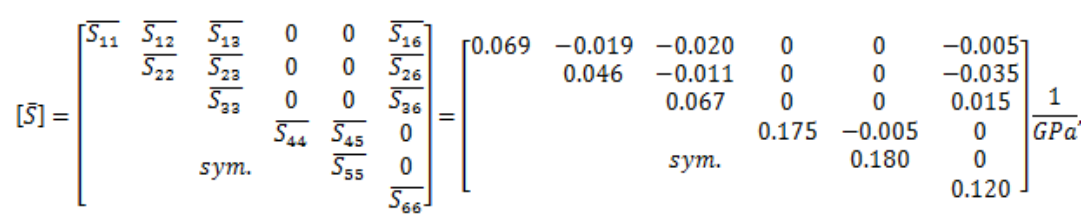

and thereby the constitutive material law in the Cylindrical coordinate system according to equation (12) results in

$$
\left.\left\{\begin{array}{c}
\varepsilon_{\tilde{z}} \\
\varepsilon_{\theta} \\
\varepsilon_{r} \\
\gamma_{\theta r} \\
\gamma_{\tilde{z} r} \\
\gamma_{\tilde{z} \theta}
\end{array}\right\}=\left[\begin{array}{cccccc}
0.069 & -0.019 & -0.020 & 0 & 0 & -0.005 \\
& 0.046 & -0.011 & 0 & 0 & -0.035 \\
& & 0.067 & 0 & 0 & 0.015 \\
& & & 0.175 & -0.005 & 0 \\
& & \text { sym. } & & 0.180 & 0 \\
G P a & 0.120
\end{array}\right\} \frac{1}{\sigma_{\theta}} \begin{array}{c}
\sigma_{\tilde{z}} \\
\sigma_{\theta} \\
\tau_{\theta r} \\
\tau_{\tilde{z} r} \\
\tau_{\tilde{z} \theta}
\end{array}\right\} .
$$


The permutation matrix $[P]$ in accordance with equation (17) and the given stress tensor in the Cylindrical coordinate system by Lekhnitskii (1963) from equation (13) follows the HoOKE's law (see equations (14) and (15))

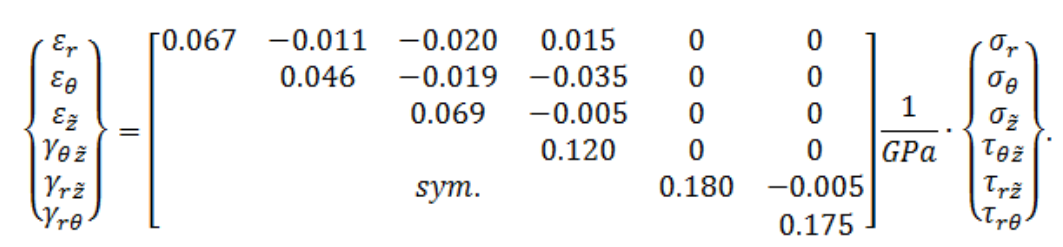

The transformed redenominated compliance matrix $[\bar{L}]$ in equation (25) under the convention of Lekhnitskii (1963) can be used to calculate the mentioned bending models.

\section{Conclusions}

With the presented method of calculation, it is possible to enter the correct material laws for the bending models of composite tubes only with the knowledge of the individual layer parameters. For this purpose, the transformed compliances which are related to the stresses and strains must not be resorted separately from Cartesian converted into Cylindrical coordinate system and reversed due to the relationships by Lekhnitskii (1963). This step will automatically be transferred by the introduced permutation matrix, which can be easily integrated for programming the analytical bending models. Therefore, calculation errors due to the inconsistent notation of the compliances are avoided and correct coordinate systems are defined which have a technical and production-related connection to fiber-reinforced plastic tubes. In comparison, Lekhnitskii (1963) refers its Cartesian coordinate system for generally anisotropic materials and does not consider the fiber orientation, which is according to its definition in the radial direction in the tube cross-section and has for this reason no technical application. Nevertheless, the relations of Lekhnitskii (1963) are necessary to use the bending models and therefore the technically meaningful coordinate system definition and transformation was presented in this publication, and the relationship for the Lekhnitskiis' (1963) definition was established with the permutation.

\section{References}

Altenbach, H., Altenbach, J. and Kissing, W. (2004). Mechanics of Composite Structural Elements. Springer-Verlag, Berlin/Heidelberg. DOI= http://bit.ly/2de RybT.

Chouchaoui, C. S. and Ochoa, O. O. (1999). Similitude study for a laminated cylindrical tube under tensile, bending, internal and external pressure. Part I: governing equations. Composite Structures, 44 (April 1999), Elsevier Science Limited, Kidlington/Oxford, 221-229. DOI= http://bit.ly/2d5Eg2S.

Derisi, B. (2008). Development of thermoplastic composite tubes for large deformation. Doctoral Thesis. Concordia University, Montréal. URL: http://bit.ly/ 2cR9azA. 
Derisi, B., Hoa, S. and Hojjati, M. (2012). Similitude study on bending stiffness and behavior of composite tubes. Journal of Composite Materials, 46 (Oct. 2012), 26952710. DOI= http://bit.ly/2cqnuZg.

Ehrenstein, G. W. (2006). Faserverbundkunststoffe: Werkstoffe - Verarbeitung - Eigenschaften [Composites: materials - processing - properties]. Carl Hanser Verlag, München/ Wien.

Ehrlich, I. (2004). Impactverhalten schwach gekrümmter Strukturen aus faserverstärkten Kunststoffen [Impact behavior of slightly curved structures of fiber reinforced plastics]. Doctoral Thesis. Universität der Bundeswehr München, München. URL: http://d-nb.info/973886102/34.

Förster, R. and Knappe, W. (1971). Experimentelle und theoretische Untersuchungen zur Rißbildungsgrenze an zweischichtigen Wickelrohren aus Glasfaser/Kunststoff unter Innendruck (Experimental and theoretical investigations of the crack initiation limit of two-layer winding tubes of glass fiber/plastic under internal pressure). Kunststoffe, 61 (1971), 583-588.

Foye, R. L. (1972). The transverse poisson's ratio of composites. Journal of Composite Materials, 6 (April 1972), 293-295. DOI= http://bit.ly/2cMcIzs.

Geuchy Ahmad, M. I. and Hoa, S. V. (2016). Flexural stiffness of thick walled composite tubes Composite Structures, 149 (Aug. 2016), 125-133. DOI= http://bit.ly/2cR8OsJ.

Jolicoeur, C. and Cardou, A. (1994). Analytical Solution for Bending of Coaxial Orthotropic Cylinders. Journal of Engineering Mechanics, 120 (Dec. 1994), 25562574. DOI= http://bit.ly/2dmvzAk.

Jones, R. M. (1999). Mechanics of Composite Materials. Taylor \& Francis Verlag, Philadelphia, PA.

Lekhnitskii, S. G. (1963). Theory of elasticity of an anisotropic body. Holden-Day, SanFrancisco, CA.

Moser, K. (1992). Faser-Kunststoff-Verbund - Entwurfs- und Berechnungs-grundlagen [Fiber-reinforced plastic - design and bases of calculation]. VDI-Verlag, Düsseldorf.

Puck, A. (1967). Zur Beanspruchung und Verformung von GFK-MehrschichtverbundenBauelementen. Teil 1. Grundlagen der Spannungs- und Verformungsanalyse [To stress and deformation of FRP multilayer composites components. Part 1. Fundamentals of stress and deformation analysis], Kunststoffe, 57 (April 1967), 284 $-293$.

Sarvestani, H. Y., Hoa, S. V. and Hojjati, M. (2016). Stress analysis of thick orthotropic cantilever tubes under transverse loading. Advanced Composite Materials, published online (June 2016). DOI= http://bit.ly/2dfObGs.

Schmidt, K. and Trenkler, G. (2015). Einführung in die Moderne Matrix-Algebra - Mit Anwendungen in der Statistik [Introduction to Modern matrix algebra - with applications in statistics]. Springer Verlag, Berlin/Heidelberg. DOI= http://dx.doi. org/10.1007/978-3-662-46773-2.

Schober, K.-U. (2008). Untersuchungen zum Tragverhalten hybrider Verbundkonstruktionen aus Polymerbeton, faserverstärkten Kunststoffen und Holz [Investigations on the behavior of hybrid composite structures made of polymer concrete, fiberreinforced plastics and wood]. Doctoral Thesis. Schriftenreihe des Instituts für Konstruktiven Ingenieurbau Bauhaus-Universität Weimar, Weimar. URL=http://bit. ly/2deRUQ3.

Schürmann, H. (2007). Konstruieren mit Faser-Kunststoff-Verbunden [Engineering with fiber-reinforced composites]. Springer-Verlag, Berlin/Heidelberg.

DOI= http://dx.doi.org/10.1007/978-3-540-72190-1. 
Shadmehri, F. (2012). Buckling of laminated composite conical shells: theory and experiment. Doctoral Thesis. Concordia University, Montréal. URL $=$ http://bit.ly/2c Maxw7.

Shadmehri, F., Derisi, B. and Hoa, S. V. (2011). On bending stiffness of composite tubes. Composite Structures, 93 (Aug. 2011), Elsevier Science Limited, Kidlington/Oxford, 2173-2179. DOI= http://bit.ly/2cWg97P.

Tarn, J.-Q., Wang, Y.-M. (2001). Laminated composite tubes under extension, torsion, bending, shearing and pressuring: a state space approach. International Journal of Solids and Structures, 38 (Dec. 2001), 9053-9075. DOI= http://bit.ly/2d5mu10.

Voigt, W. (1966). Lehrbuch der Kristallphysik [Textbook of crystal physics]. SpringerVerlag, Berlin/Heidelberg. DOI= http://dx.doi.org/10.1007/978-3-663-15884-4. 